How Fuzzy-Trace Theory Predicts True and False Memories for Words, Sentences, and Narratives

Word count: 7643

Preparation of this article was supported in part by a grant to Valerie F. Reyna from the National Institute of Nursing Research (R01NR014368-01) and the National Science Foundation (SES1536238).

Correspondence concerning this article should be addressed to Valerie F. Reyna, Human Neuroscience Institute, G331, Martha Van Rensselaer Hall, Cornell University, Ithaca, NY

14853. E-mail: vr53@cornell.edu 


\begin{abstract}
Fuzzy-trace theory posits independent verbatim and gist memory processes, a distinction that has implications for such applied topics as eyewitness testimony. This distinction between precise, literal verbatim memory and meaning-based, intuitive gist accounts for memory paradoxes including dissociations between true and false memory, false memories outlasting true memories, and developmental increases in false memory. We provide an overview of fuzzytrace theory, and, using mathematical modeling, also present results demonstrating verbatim and gist memory in true and false recognition of narrative sentences and inferences. Results supported fuzzy-trace theory's dual-process view of memory: verbatim memory was relied on to reject meaning-consistent, but unpresented, sentences (via recollection rejection). However, verbatim memory was often not retrieved, and gist memory supported acceptance of these sentences (via similarity judgment and phantom recollection). Thus, mathematical models of words can be extended to explain memory for complex stimuli, such as narratives, the kind of memory interrogated in law.
\end{abstract}

Keywords: fuzzy-trace theory; false memory; development; gist; narrative memory 
This article provides a review of fuzzy-trace theory's (FTT) theoretical framework that unifies and refines constructivist and information-processing approaches by relying on a distinction between literal, precise memory representations (verbatim traces), and vague, meaning-based memory representations (gist traces), both of which improve with development (Brainerd \& Reyna, 2005; Reyna, Wilhelms, McCormick, \& Weldon, 2015). In this review, we discuss how verbatim and gist memory accounts for dissociations between true and false memory, distinguishing spontaneous false memory (e.g., people misremember because they make inferences) from misinformation effects (e.g., people misremember because interrogators introduce false information). We then explain how seemingly paradoxical findings in false memory that are not easily accounted for by associationist theories of memory (e.g., Gallo \& Roediger, 2003), such as the greater persistence of false as compared to true memory, are predicted by fuzzy-trace theory.

We argue that artificial distinctions between studies of true memory as opposed to false memory are no longer tenable. Indeed, accounts of memory that exclude consideration of meaning-related "false" memories are not only incomplete, they are misleading. To illustrate, true recall can be based on readout from verbatim memory or reconstruction from gist memory (followed by a familiarity judgment). For example, given the following study list - bed, rest, dream, snooze, blanket, doze, slumber, snore, nap, peace, yawn, and drowsy- people may correctly recognize "snooze" on a subsequent recognition task because they retrieve a verbatim trace of the presented word, or they could have inferred its presence based on the overall gist of the list as one containing sleep-related terms. Thus, even correct recall (e.g., of "snooze") is not a pure measure of a single memory process, but, rather, has been shown to be produced by dual verbatim and gist processes (e.g., Brainerd, Reyna, \& Howe, 2009). Therefore, directly 
comparing true and false memory — as is done routinely in behavioral and functional magnetic resonance imaging (fMRI) studies — does not necessarily isolate memory processes. People can testify accurately about their memory for events either because they retrieve verbatim memories or because they reconstruct them from gist. The accuracy of the memory report does not unambiguously prove its psychological origin.

Knowing the conditions under which memories were encoded and tested sometimes makes it possible to infer their psychological origin (Reyna, Mills, Estrada, \& Brainerd, 2007). In this article, we report the results of an experiment about memory for narratives that illustrates many of these conditions and their effects on memory. Narrative memory, as opposed to memory for words, more closely resembles memory for witnessed events. One such condition is the delay between the witnessed event and testimony about the event. Eyewitness reports that occur immediately after a witnessed crime are more likely to be based on verbatim memory than reports given later in court and, thus, are more likely to be accurate about arbitrary details. However, a legal case often does not turn on arbitrary details. Later testimony in court that requires an inference, for example about whether the accused was acting strangely or was angry on the day of the crime, draws on memory for the gist of events, and such gist generally endures over long delays. The gist of an event is not random and, based on knowledge about the witness (e.g., a rival gang member) and circumstances (e.g., the accused was standing over the decedent and, hence, is remembered as holding the gun), likely hypotheses can be generated about the type of information the witness would be likely to infer. These hypotheses can then be tested with other evidence.

Therefore, there are two kinds of accuracy that are relevant in the real world and that must be distinguished: literal verbatim accuracy and substantive accuracy about the gist of 
events. A witness can be right about the gist of an event (e.g., that they were robbed or molested), but wrong about details (the color of a shirt or a car), and vice versa. We use the term "true" memory in its traditional sense in the literature to refer to memory for actual events as they were experienced (i.e., verbatim memory), as contrasted with "false" memory which can be substantively accurate or not. The law assumes that a witness's memory for actual events can be absent, weak, or mistaken, but, when witnesses claim to remember, they are supposed to testify only to what they have experienced. The law fails to accommodate the fact that people routinely "remember" a gist of events that they never experienced.

The accuracy of memory reports not only depends on the quality of verbatim and gist memories, but also on how questions about memory are asked. For example, memory reports are influenced by recognition probes: Asking witnesses about an event that really happened can trigger verbatim memories, but asking them about a true inference (e.g., an event they know to be true but could not have been directly observed) can elicit memories for the gist of the event. Fortunately, these ambiguities in memory reports have been addressed in research that we now discuss, which helps to clarify otherwise mysterious memory phenomena, including dissociations between true and false memory, false memories outlasting true memories, and developmental increases in false memory.

\section{Dual Processes in Memory}

\section{What is false memory?}

Studies of autobiographical and everyday episodic memories have revealed that a surprising number of real-life "memories" are false in the sense that they were never experienced (Frenda, Nichols, \& Loftus, 2011). Instead, memories can be suggested (e.g., by viewing family photographs), imported from one context in which they did occur to another in which they did 
not occur (source misattribution e.g., when events from a movie are remembered as personally experienced), or, most commonly, they can represent semantic and inferential extensions of experienced events (e.g., seeing the accused carrying a shirt into a laundry room and hearing a washing machine turn on in that room soon afterwards can be misremembered as having seen the accused put the shirt into the washer; Brainerd, Reyna, Holliday, \& Nakamura, 2012; Ceci \& Bruck, 1993; Dodson \& Shimamura, 2000). Laboratory research has focused mainly on two paradigms: misinformation in which never-experienced events are suggested (e.g., through suggestive questions or direct misinformation) and spontaneous false memories that occur as a result of ordinary processes of understanding and inference, namely, gist extraction (for a more complete taxonomy of types of false memory, see Table 17.1 in Reyna, Mills, Estrada, \& Brainerd, 2007).

A popular false-memory paradigms is the Deese-Roediger-McDermott (DRM) task in which presented lists consist of words that share numerous semantic relations with one another (e.g., bed, rest, dream, snooze...), relations that go beyond mere word association or contiguity frequency (Brainerd, Yang, Reyna, Howe, \& Mills, 2008; Robinson \& Roediger, 1997). In recognition tests, presented words and unpresented but semantically related distractors (e.g., sleep) are probed, as well as unrelated distractors that do not share meaning with presented words (e.g., apple). This paradigm typically produces high levels of recognition for the unpresented distractors that are semantically related to multiple words on the list, often rivaling the acceptance rate for presented words (Reyna \& Lloyd, 1997).

We concentrate on spontaneous false memory for semantically related words and sentences because these paradigms most clearly reflect gist-based processes in memory falsification. Recent studies explain why this is so; such studies have failed to find correlations 
between misinformation effects (when subsequently presented misinformation is reported as having occurred as part of an original event) and false memories for semantically related word lists, referred to as DRM effects (e.g., Ost, Blank, Davies, Jones, Lambert, \& Salmon, 2013). FTT predicts that both misinformation and DRM effects are products of verbatim and gist memories (Barnhardt, Choi, Gerkens, \& Smith, 2006; Reyna \& Titcomb, 1997; see Brainerd, Reyna, Wright, \& Mojardin, 2003, and Mojardin, 1998, for parameter estimates supporting this prediction). However, verbatim memory (for the misinformation) promotes false-memory acceptance in the misinformation paradigm, whereas it promotes false-memory rejection in the DRM paradigm. Because misinformation is typically gist-consistent in experiments, gist memory promotes false-memory acceptance in both paradigms.

To illustrate these relationships, consider the following example: Imagine that an interrogator inadvertently introduces misinformation that a perpetrator had a hammer (when he actually had a screwdriver). The better that witnesses remember the presented word "hammer" from the interrogation, the more likely they are to later accept the false statement that the perpetrator had a hammer when questioned in the courtroom (positive correlation). (Misinformation effects occur when people are more likely to remember the manipulated information presented in the interrogation than the information from the original event.) However, in the DRM paradigm, better verbatim memory for the presented word "screwdriver" would help people reject the unpresented word "hammer" (negative correlation). Remembering the gist that there was a tool of some kind would support acceptance of either hammer or screwdriver in both the misinformation and DRM paradigms (e.g., gist supports positive correlations between accepting "tool" and both "hammer" or "screwdriver"). This confluence of conflicting relationships can yield an overall lack of correlation between misinformation (e.g., 
when based on verbatim memory) and DRM effects (e.g., when based on gist memory), explaining the results of Ost et al. (2013). Therefore, although misinformation is more effective in falsifying memory when it is plausible (i.e., when it is consistent with the gist of experienced events) as opposed to implausible, misinformation effects also tap verbatim memories — making these false memories less suitable for demonstrating effects of meaning in the real world. Thus, we focus on false memories that reflect meaning-making, rather than misinformation effects, because of the broad applicability of meaning in applied contexts, such as the law.

\section{How does FTT explain false memories?}

In the DRM paradigm, subjects see or hear lists that include the first 15 word associates for a given "critical" lure word and are then given a recall or recognition test (for reviews, see Brainerd \& Reyna, 2005; Gallo, 2006). The unpresented critical lure word is falsely remembered at very high levels, almost as highly as presented targets. Presented words are called "targets" because the standard DRM paradigm involves a verbatim test: Subjects are instructed to say "yes" only to exact copies of presented words, not similar or related words. Early explanations of the DRM phenomenon focused on associative activation of lures by target words, either at study or test (Deese, 1959; Underwood, 1965).

In the modern era, since Roediger and McDermott's (1995) re-introduction of the paradigm, FTT was the first theory applied to the DRM effect (e.g., Reyna \& Lloyd, 1997; Robinson \& Roediger, 1997; see also "false memory" in Reyna \& Brainerd, 1995a). According to FTT, verbatim and gist memory influence false alarms when people misrecognize related distractors (e.g., unpresented critical lures), although the influence flows in opposite directions: verbatim memories are used to reject related distractors (remembering "doze," "snooze" etc. produces rejection of "sleep"), but gist memory is used to accept related distractors 
(remembering that the gist or theme of the list was sleep produces acceptance of sleep), making FTT an opposing processes theory of false memory.

Conceptually-related distractors (e.g., "sleep") are good cues for gist memories (which produce acceptances, as in false recognition), and they are also better cues for verbatim memories (e.g., putting "sleep" on the test can remind people of "snooze" from the study list) than unrelated distractors are (e.g., putting "apple" on the test does not remind people of the sleep-related study items). When related distractors are tested shortly after the study list is presented, they can cue retrieval of verbatim memories. (Unrelated distractors-being unrelated to the studied items - do not remind people of the studied items.) Retrieving verbatim memories then produces rejections, called false-recognition reversal because related distractors are rejected more often than unrelated distractors (e.g., Brainerd, Reyna, \& Kneer, 1995). As predicted, false-recognition reversal occurs when verbatim memories are accessible, as when delays between study and test are short or when the same (verbatim) targets have been studied repeatedly. Thus, theoretically expected variations in the contributions of gist and verbatim memories both determine responses to related distractors.

In addition, FTT distinguishes verbatim memory from metacognitive monitoring and response inhibition; response inhibition is captured in response bias parameters in recognition models (Reyna \& Brainerd, 1998; see also Klaczynski, 2009). (Only parameters required by data are included in FTT models.) Metacognitive monitoring depends on such cues as phenomenology (see Johnson, Hashtroudi, \& Lindsay, 1993). Thus, phenomenology—the conscious experience of remembering as vague or vivid--is distinguished from memory representations per se (Reyna, Holliday, \& Marche, 2002). Verbatim representations are experienced as vivid and gist representations as vague, except when gist is repeatedly cued. 
Strengthening gist memories, for example, through repeated presentation of different target words that cue the same meaning, ultimately produces vivid phenomenology (e.g., highconfidence false memories characterized as "remembered" as opposed to merely "known"), called phantom recollection to distinguish it from vague gist-based similarity. However, phantom recollection behaves like gist-based similarity; it is stable over a delay (verbatim recollection dissipates with delay) and it loads with other gist parameters in factor analyses (verbatim-based recollection of targets and recollection rejection of distractors also load together, but separately from gist parameters; Brainerd, Wright, Reyna, \& Mojardin, 2001).

As we presently discuss, FTT applies analogous constructs to explain false memories for words (e.g., the DRM effect), sentences, and narratives. However, it is important to acknowledge alternative accounts. In 2001, Roediger et al. introduced the activation-monitoring account of the DRM effect, another opposing-processes approach, but one that conflates monitoring with true memory (see Roediger, Watson, McDermott, \& Gallo, 2001). Associative activation continues to have its proponents (e.g., Howe, 2008), despite the evidence from the cognitive revolution demonstrating that many aspects of cognition cannot be explained by associative activation (e.g., Mandler, 1962). For example, Tolman (1948) reported that even lowly rats initially trained in a T-maze to turn in a specific direction to acquire food (e.g., left) were able to turn in a different direction to find the food when the maze was rotated or the original route was blocked -- suggesting that the rats relied on cognitive maps rather than rote stimulus-response associations (see also Mayer, 2002). Associative activation remains an appealing idea because it seems straightforward and easy to operationalize (e.g., word association norms are extensive and freely available; Nelson, McEvoy, \& Schreiber, 2004). 
However, as we discuss in the next section, the evidence overwhelmingly favors gist $—$ or meaning — as the main source of spontaneous false memories.

\section{Spontaneous False Memories are Mainly Based on Meaning, Not on Meaningless}

\section{Associations}

Associationism remains a popular account of human memory and its main ideas can be illustrated using the DRM paradigm (e.g., Schacter, Guerin, \& St. Jacques, 2012). The strength of associations between words is highly correlated with meaning: Associated words share meaning and words that share meaning are associated with each other (Brainerd, Yang, Reyna, Howe, \& Mills, 2008; Hutchison \& Balota, 2005). However, false memories display a number of characteristics that rule out associative activation as their sole or major cause. The most notable characteristic is that false memories persist over time (even when response bias is accounted for). Stability over time is consistent with FTT's explanation that gist underlies false memory (because gist is a relatively stable form of memory). Going back to our previous word list (bed, rest, dream, snooze...), a person may incorrectly recall that "sleep" was on the list. If the person is asked to recall the word list a month later, he or she may only recall the gist of the list -that the list contained sleep-related words -and therefore be more likely to falsely recall "sleep" (a superior exemplar) than a less representative word such as "rest." In contrast, associative activation—also referred to as priming—is short-lived, dissipating within minutes as opposed to persisting for days and weeks (and longer) as false memories do. The stability of false memories over time is a consistent result that has been replicated in scores of studies (see Gallo, 2006; 2010, for reviews).

In addition, if false memories occurred because target words activated lures either at study or test, there would be positive correlations between true and false memory. Instead, true 
and false recognition in the DRM paradigm are generally independent of one another, and true and false recall correlate negatively, again, a robust finding across studies and one that is predicted by FTT under typical DRM testing conditions (e.g., Brainerd et al., 2008; Roediger et al., 2001). At a minimum, associative activation accounts that lack a countervailing rejection process can be ruled out because a negative correlation requires a process that opposes acceptance of false memories. Also, as noted above, true and false memory measures load on orthogonal factors in principal component analyses (see below).

If the dual processes that account for false memories are assumed to be (a) memory for presented words (working against false memories, called recollection rejection) and (b) memory for the shared meaning of presented words (working to promote false memories), such an account simply reduces to FTT's. However, FTT's explanation is not post hoc; it is supported by independent evidence from research on psycholinguistics, memory, gestalt theory, and judgment and decision making. FTT can be thought of as combining the schema-theory (gist-like memory) and verbal-learning (verbatim-like memory) traditions, but without many of the empirical and theoretical problems associated with those approaches (e.g., Alba \& Hasher, 1983). In fact, FTT was based directly on the psycholinguistic literature of the 1980's, and accounts for findings such as those from Spiro (1980), who found that experimental subjects retrieved verbatim traces when instructed to recall precise passages from a text and gist traces when they were instructed to make meaningful inferences.

Associative activation of lures by targets is an intuitively appealing explanation of DRM effects - after all, the lists are constructed by selecting words that are associates of the lures. Association refers literally to the tendency for one word to evoke another-reflecting stimulusstimulus association in everyday experience (Nelson et al., 2004; Tolman, 1938). (Association is 
also used to explain memory for more complex stimuli and has been called connectionism and binding; Schacter et al., 2012; Schneider \& Anderson, 2012). However, scoring the 15 targetlure word pairs for semantic properties for each of 55 DRM lists (825 pairs), Brainerd et al. (2008) showed that fifteen semantic properties varied reliably with forward or backward association between targets and lures. This result makes sense because association in everyday experience is not arbitrary-words co-occur in meaningful contexts. Hence, associative activation is a marker (or correlate) of meaning — not necessarily a causal mechanism.

Our arguments so far do not suggest that associative activation does not exist under any circumstances. In fact, there is good evidence that it does exist (see Hutchison \& Balota's, 2005, discussion of "semantic" priming which is apparently lexical, not semantic). That is, words can be primed (i.e., associatively activated) in a manner similar to a parrot repeating the phrase "Polly wants a cracker" in response to a cue-without understanding or gist. However, that lexical priming fades away quickly, and so does not characterize the usual DRM effect which endures over a delay (Meade, Watson, Balota, \& Roediger, 2007). Showing that gist processing was especially influential after a delay, Huff, McNabb, and Hutchinson (2015) conducted a series of critical tests of activation monitoring versus FTT, which supported FTT.

Furthermore, consistent with FTT's dual-process account, Brainerd et al. (2008) also showed that variability across the 55 lists in false recall, false recognition, and backward associative strength loaded on a single semantic factor (familiarity/meaningfulness), whereas variability in true recall loaded on an independent factor (imagery/concreteness). With respect to the latter factor, images, and words that evoke images, exhibit superior verbatim memory (Paivio, 1971). The verbatim advantage for images or pictures is not the same thing as the distinctiveness heuristic, a metacognitive strategy (Schacter, Israel, \& Racine, 1999). 
Perhaps the strongest evidence for the semantic basis of false memories (and against rote association), Cann, MacRae, and Katz (2011) demonstrated in three experiments that semantic properties — in the absence of forward or backward associations - were sufficient to elicit gist meanings and produce false memories. Specifically, Cann et al. (2011) provided word lists that revolved around a situational theme (e.g., luggage, passport, chairs, runway -all meaningfully related to the critical word airport). Crucially, these words were not associatively related (i.e., people did not tend to think of these words when asked about the first word that came to mind when given the word airport). The fact that false memories were found for lists that elicited a meaningful gist but were not associated provided a critical test of FTT, demonstrating the importance of gist to false memory creation.

\section{Do these conclusions that gist underlies false memories for word lists explain more complex stimuli?}

DRM word lists emulate an important feature of everyday experience, namely, connected meaning that revolves around a semantic theme (Brainerd, Reyna, \& Zember, 2011; Reyna, Mills, Estrada, \& Brainerd, 2007). People are more likely to attend to information that is meaningful (i.e., has semantic content; McElroy, Dickinson, Corbin, \& Beck, 2015; New \& German, 2014). Although DRM stimuli provide a convenient method for measuring falsememory, it is also important to determine whether the findings for word lists can translate to more complex stimuli (e.g., narratives), the kind of memory interrogated in applied contexts such as law.

The same concepts--and the same models--that we have identified in DRM research also apply to research on connected meaning in units larger than words, such as narratives (e.g., Brainerd, Reyna, \& Estrada, 2006; Britt, Kurby, Dandotkar, \& Wolfe, 2008; Fisher \& Chandler, 
1991; Reyna \& Kiernan, 1994, 1995). For narratives, sentences constitute the "dots" that support a more global semantic representation or theme, just as words make up the "dots" that support the overall theme of a semantically associated word list. Whether it is reading a blog, listening to evidence in a trial, or merely connecting the dots of ordinary experience, people interpret stimuli and make inferences that go beyond the literal information (Hans \& Reyna, 2011; Pennington \& Hastie, 1992).

Psycholinguistic research showed that multiple levels of gist could be identified for a single narrative (e.g., text and situational models), encoded along with the verbatim content (Clark \& Clark, 1977; Glucksberg \& Danks, 1975). The gist of narratives was measured using multiple methods that converged in their results - such as asking subjects to summarize the main points of a narrative or to recall it after a delay, or by analyzing the propositional structure of a narrative and identifying the nodes at the top of the structure (Kintsch, 1975). When considering whether there is sufficient evidence for gist or whether there are objective methods to measure it, it is useful to note that there is a the vast literature on psycholinguistics that dates back to the 1970's (and beyond). We rely on the details of that highly rigorous literature in FTT, although we have extended the original ideas in key respects (see Reyna, 2012; Reyna \& Brainerd, 1995b).

Specifically, FTT extends prior psycholinguistic theory in assuming, as research from many laboratories has demonstrated, that verbatim and gist representations are independent, which explains a host of paradoxes and contradictions. Independence implies that different measures of "memory" (that tap verbatim and gist memory differentially) can be unrelated, positively related, or even go in opposite directions under predictable conditions. As examples of these relationships between different measures of memory, deep levels of processing increase 
both true (accuracy increases) and "false" (accuracy decreases) recall, performing an initial verbatim (word-by-word) translation fails to facilitate subsequent gist summaries of the same material and, under speeded-response conditions, false recognition initially increases (as the time allowed for a response lengthens) but then changes direction and decreases (Brainerd, Reyna, Wright, \& Mojardin, 2003; Michael, Allison, Danks, de Terra, Massaro, \& Donavos, 2007; Thapar \& McDermott, 2001; Toglia, 1999). Each of these seemingly contradictory relationships is easily explained using FTT and requires a distinction between verbatim and gist memory. Respectively, deep processing promotes gist which increases true and "false" gist-based memory; verbatim translation is independent of gist-based summarization; and recognition is initially gist-based (promoting "false" recognition) and then verbatim-based (promoting rejection of unpresented gist-based "false" items). In fact, such demonstrated dissociations are required to assert that there are dual processes (Keren \& Schul, 2009).

In particular, we now discuss detailed psycholinguistic findings from an experiment that provide evidence for such dual verbatim and gist representations in narratives (Lim, 1993: see also Brainerd et al., 2006; Brainerd \& Mojardin, 1998; Gerkens \& Smith, 2004; Reyna \& Kiernan, 1994; Singer \& Remillard, 2008). Although there is support in the psycholinguistic literature for FTT, the following experiment unconfounds factors that are typically confounded in that literature (e.g., surface similarity of paraphrases and inferences to originally presented sentences) and its design allows estimation of underlying memory processes using mathematical models. Thus, this experiment illustrates how FTT's predictions can achieve the laudable precision of word-list experiments with the broader applicability of memory for narratives.

Forty-eight students at a large southwestern university were presented with eight narratives (presented in a different random order for each subject), such as "The bird is inside the 
cage. The cage is under the table. The bird has yellow feathers." Subjects were tested twice, once after a buffer task of about 30 minutes and again after a 12-day delay. Test sentences varied with respect to three factors in a 2 (true/false) X 2 (premise/inference) X 2 (old/new) design; both presented sentences (the premises that support inferences) and the filler sentence ("The bird has yellow feathers.") were also tested, yielding 10 test sentences in all. That is, the true $(\mathrm{T})$ premise $(\mathrm{P})$ sentences with old words $(\mathrm{O})$ were presented sentences (designated TPO). Inferences (designated as I) differed from premises by involving new combinations of words. New combinations of old words could be true ("The bird is under the table," TIO) or false ("The table is under the bird," FIO.). Thus, true sentences were consistent with the gist of presented sentences, and new sentences differed in one or two verbatim (surface) features from presented ones. Because of the factorial design, each true sentence had a corresponding false sentence with the same surface features (i.e., TPO-FPO, TIO-FIO, TPN-FPN, and TIN-FIN).

One half of the subjects was randomly assigned to a verbatim-acceptance memory condition (i.e., old-new recognition) and were instructed to try to "remember the story" and told that they would be asked to say "yes" only to those sentences that were exactly the same. This verbatim-acceptance condition is like being a witness in a courtroom (Reyna et al., 2007). A witness is required to testify only to what he or she personally experienced, rather than about hearsay or inferences. It is then up to the triers of fact, juries or judges, to make inferences. The distinction between verbatim memory and inferences is more than academic; it is easy to imagine the miscarriages of justice that would result if witnesses could testify to their beliefs as though they were witnessed, as opposed to being limited to testifying about memories of direct experience (Faigman \& Monahan, 2005). 
In most situations in real life, however, people are expected to report the gist of experience when they are asked about their memories. Medical students, for example, must remember the clinical gist of patients' symptoms (learning to pick up the theme or gist that connects symptoms) to properly diagnose patients (Lloyd \& Reyna, 2009). The other half of the subjects was assigned to this kind of situation, a gist-acceptance meaning condition, and told to think about "what the story means" and to say yes to according to "what was true" or "what was meant." Crucially, instructions for each group included examples of true inferences that should be rejected in the memory (verbatim acceptance) condition and accepted in the meaning (gist acceptance) condition, along with presented and false sentences. It turns out that very high rates of false recognition of sentences can occur because subjects misinterpret the task; remembering "exactly" what happened ordinarily refers to memory for gist in everyday situations (Reyna \& Kiernan, 1994). Indeed, the U. S, Supreme Court has ruled that journalists need only capture the gist when quoting sources - quoted statements do not have to be verbatim. Thus, it is important to provide examples of true inferences that must be rejected to make the verbatim-acceptance task clear and to control for familiar wording, as done here; otherwise, the results are misleading (cf. Bransford \& Franks, 1971).

Although the verbatim- and gist-acceptance conditions emulate real-world memory situations, their greater utility lies in being able to disentangle the hidden contributions of different kinds of memories. In particular, these two conditions by themselves make it possible to identify (and estimate) processes that counteract false memories and those that promote them (e.g., Brainerd \& Reyna, 2002; Singer \& Remillard, 2008; Stahl \& Klauer, 2008).

As Figure 1 shows, although the sentences in stories did not repeatedly instantiate the same gist (as DRM lists do), which drives up false memories, false memory effects can be 
detected by comparing the darker bars for corresponding true and false pairs of sentences. True but never-presented sentences were accepted significantly more often than similarly worded false sentences in the verbatim-only condition. The meaning condition is shown in lighter bars for comparison. However, as we have discussed, false recognition is a function of countervailing processes: verbatim-based recollection rejection, gist-based acceptance, and response bias that typically varies across verbatim-acceptance (T or target-only instructions) versus gist-acceptance conditions ( $\mathrm{T}+\mathrm{R}, \mathrm{R}$ for related items, instructions; see Brainerd et al., 2006). It is important to distinguish memory processes from response bias to better understand how witnesses could carefully monitor their testimony (i.e., be highly motivated to adopt a strict response criterion) and, yet, still report false memories.

Fortunately, we can use established modeling procedures based on FTT to estimate each of these processes from recognition data such as that shown in Figure 1. Applying Equations 12 and 13 from Brainerd and Reyna (2002) to estimate parameters, the probability of verbatimbased recollection rejection is higher for the inferences that differ in two surface features from presented sentences (TIN-FIN) compared to inferences that differ in only one surface feature (TIO-FIO), .48 versus .38 for recollection, respectively. Conversely, the probability of gistbased acceptance (subsuming both gist-based similarity and phantom recollection) is higher for similar inferences (TIO-FIO) than dissimilar ones (TIN-FIN; .29 vs. 15, respectively). Thus, the higher observed levels of false recognition for more similar true inferences derives from two independent memory sources - lower verbatim-based rejection and higher gist-based acceptance (controlling for response bias).

Although the parameter estimates are independent for gist and verbatim memories, the observed probability of gist-based acceptance is contingent on verbatim memory-which is used 
first, if it can be remembered, to either accept (in the meaning condition) or reject (in the memory condition) unpresented true sentences. Therefore, the overall probability of accepting a distractor based on meaning in the meaning-acceptance condition (by remembering the presented true sentences or their gist) is .56 for both types of inferences (with the remaining proportion of .20 "yes" responses due to bias in the meaning condition, for an overall acceptance rate of .76).

Response bias differs in the usual direction between the memory (average beta $=.34$ ) and meaning (average beta $=.48$ ) conditions, greater bias toward saying "yes" when more sentences (verbatim and meaning-consistent) can be accepted, which is generally true in experiments with DRM lists, other words lists, and sentences (Brainerd \& Reyna, 2008). People say “yes" more often when more types of items are acceptable, an interesting effect in its own right (Dobbins, Kroll, \& Liu, 1998).

Remarkably, the effect of meaning bled through even in the verbatim-only condition, although narratives were carefully controlled for plausibility (so that subjects were forced to remember the presented stories rather than rely on general knowledge, as they often can in real life); subjects had above-average intelligence and educational levels; and they were warned that they would have a memory test (intentional memory is known to be higher than incidental memory; Hunt \& Ellis, 2004). Furthermore, delayed memory performance was enhanced by a prior memory test (e.g., Roediger \& Karpicke, 2006), which revives fading verbatim memories of presented items (though it also gooses false recognition of previously tested related distractors; Reyna \& Brainerd, 1995a; Reyna \& Titcomb, 1997). In spite of these factors that increased reliance on arbitrary episodic memories, the systematic influence of gist representations of sentences could be discerned. Thus, despite ideal conditions that are rarely 
present in real life, people falsely remembered the meanings of narratives as though they had been experienced.

However, these findings of meaning-based false memories do not imply that verbatim memories are necessarily distorted, overwritten, or forgotten. Instead, FTT assumes that verbatim and gist memories are encoded, stored, and retrieved independently (e.g., Reyna \& Brainerd, 1995a). Evidence for the independence of verbatim and gist memory is provided by examining the relationship between judgments of presented sentences and gist-consistent distractors (e.g., true inferences). This relationship was evaluated in the experiment described above as well as in other experiments. FTT predicts that presented sentences that are tested after a short delay under verbatim-acceptance instructions (say yes only to sentences that were presented) are accepted mainly using verbatim memory, but true paraphrases or inferences are accepted using gist memory. Therefore, according to FTT, acceptances of presented sentences and true inferences should be independent because verbatim and gist memory are independent, which has been found in multiple experiments, contradicting predictions of schema theory or constructivism (e.g., Brainerd \& Mojardin, 1998; Lim, 1993; Reyna \& Kiernan, 1994; Singer \& Remillard, 2008). This relationship between verbatim and gist memory is assessed through very sensitive likelihood ratio tests, which compare the unconditional probability of saying yes to a true paraphrase or inference (gist memory) to the conditional probability of saying yes to a true paraphrase or inference given that they said yes to the presented sentence for that story (verbatim memory). Combining many memory studies by different authors reveals no increase or decrease in the conditional probability relative to the unconditional probability, supporting independence of verbatim and gist memory (e.g., Brainerd \& Reyna, 1992; Fisher \& Chandler, 1991; Reyna \& Brainerd, 1995a). What this means is that there is no relationship between saying yes to the true 
paraphrases or inferences (i.e., saying that these unpresented sentences were presented) based on gist memory and saying yes to the actually presented sentences based on verbatim memory.

However, after time passes, verbatim memories become less accessible. Even in the verbatim memory condition, after a long delay (more than a week), recognition of any true sentence — presented or unpresented — is mainly based on gist (e.g., Goldsmith, Koriat, \& Pansky, 2005). Hence, again based on FTT, acceptances by the same subjects of the same presented sentences and true paraphrases or inferences should become positively dependent after such a delay, which was also found (Lim, 1993; Reyna \& Kiernan, 1994).

In contrast, under meaning instructions, presented sentences are recognized using gist from the outset, and so their acceptance is positively related to acceptance of other true sentences at both shorter and longer delays. Finally, again, as predicted by FTT, boosting verbatim memory for presented items (e.g., through repetition of exact wording at study, testing presented items immediately prior to true distractors at test, or using distinctively worded metaphorical sentences) produces negative dependency at shorter delays under verbatim-acceptance instructions (Brainerd, Reyna, \& Kneer, 1995; Reyna \& Brainerd, 1995a; Reyna \& Kiernan, 1995). Boosting verbatim memory allows subjects to reject true distractors more effectively, using recollection rejection.

Two crucial caveats should be mentioned to correctly interpret the results of tests of verbatim-gist independence: a) recognition is not based purely on either verbatim or gist memory in any condition (so relationships between sentences are probabilistic not deterministic) and, moreover, b) relationships between sentences must be computed within each story to test hypotheses about independence. That is, we examined the relationship between memory for presented sentences and "memory" for the true inferences that followed from those same 
presented sentences. If acceptances of different kinds of sentences were merely totaled within conditions, these totals would reflect individual differences (e.g., in metacognitive strategies, inhibition, or intelligence) rather than be tests of the relationship between verbatim and gist memory per se; within-story dependency assessment is a more stringent test of dissociated memory systems. More generally, true memories and "false" memories (for inferred or meaning-consistent gist) for the same event are distinct: The same event can be remembered in two ways - one true and the other false. Thus, the findings of verbatim-gist independence argue against constructive (or schematic) theories of memory, which claim that the constructed memory distorts or overwrites original memories. In police interviews and court testimony, the possibility of distinct memories can be pursued in interrogations to ferret out original memories despite vivid false memories of gist.

\section{Developmental Reversals in Memory}

If verbatim and gist representations form the basis of independent systems of encoding, storing, and retrieving memories, then it follows that they could develop independently across the lifespan (Reyna, 2011). In other words, verbatim and gist memories need not develop in lockstep or at similar rates. Consistent with this hypothesis of independence, Reyna and Kiernan (1994) reviewed evidence of developmental reversals in memory in which systematic departures from accuracy increased with age from childhood to adulthood. These increases generally reflected the growth of increasingly sophisticated reasoning that colored memory for stories and other meaningful stimuli (e.g., Liben, 1992). The developmental-reversal effect was empirically inconsistent, however. The key to Reyna and Kiernan's analysis of the literature is that it laid the groundwork for FTT's predictions about the conditions under which false memories should increase versus decrease with age. 
As expected based on FTT, memory sometimes showed developmental progression (traditional age-related improvement), and sometimes developmental reversal, in memory for stories. In line with the parallels between word lists and stories that we have discussed, Brainerd, Reyna, and Forrest (2002) used FTT to generate paradoxical predictions for DRM lists. They showed that both verbatim and gist memories for DRM lists increased from childhood to adulthood, but the increase was sharper for gist than verbatim. Hence, the net accuracy of children was higher than that of adults. This predicted developmental reversal effect - an increase in false memory from childhood to adulthood - has been replicated in 53 of 55 DRM experiments since 2002 (Brainerd et al., 2011).

Moreover, developmental increases in false memory exhibit telltale signs that they are gist-based (without sufficient opposition from verbatim memory). To take a couple of examples, intrusions in free recall become increasingly semantic with age during childhood. Younger children have intrusions in recall but these are typically not semantically related to the theme of the DRM lists, even when those lists are normed so that the younger children understand each word. Semantic clustering in recall also increases during childhood, and the amount of such clustering is related to the amount of false memory. Response bias simultaneously decreases in this period, and seemingly contradictory developmental trends can be brought into harmony with the finding of developmental reversals by taking that into account (e.g. Ghetti, 2008). During interviews with children, then, it is useful to anticipate that younger children are more likely to agree with interrogators (because of a yea-saying bias, especially to authority figures), but they are less likely to generate gist-based false memories of events.

Developmental reversals have also been obtained for stories, explained in terms of verbatim versus gist memories (Ceci, Papierno, \& Kulkofsky, 2007; Reyna \& Brainerd, 2011; 
Reyna \& Kiernan, 1994). At the same time, the verbatim-gist distinction explains why other false-memory effects, such as the misinformation effect, would decrease with age. Briefly, as we discussed earlier, in experiments on misinformation, such effects tap verbatim memory for introduced misinformation--sometimes about arbitrary details - and the typical misinformation experiment does not include the repetition (and strengthening) of gist that characterizes the DRM effect. Conversely, when original verbatim information is repeatedly tested, this testing inoculates against effects of later misinformation (Pansky \& Tenenboim, 2011. The point is that effects of verbatim and gist memory can be clearly separated, and they combine to create developmental effects.

To illustrate differential effects of gist and verbatim memory on developmental reversals, consider manipulations that boost reliance on gist — assisting children in spontaneously "connecting the dots" and recognizing semantic themes. The most straightforward of these manipulations is to encourage children to search for meaningful relations as stimuli are encoded, called “gist cuing” (Reyna \& Kiernan, 1994). Holliday, Brainerd, and Reyna (2008) introduced gist cuing with DRM lists and found that it increased false recall, and the increase was greater at intermediate ages (e.g., 13) than at earlier (e.g., 9) or later (e.g., 15) ages , as expected. Bridging DRM lists and narratives, Dewhurst, Pursglove, and Lewis (2007) as well as Howe and Wilkinson (2011) presented words from DRM lists in stories that revolved around the themes of the lists. Stories increased false memories in younger children, compared to standard presentation of DRM word lists, but had little effect on older children. Thus, developmental reversals were more pronounced with standard presentation than with narratives that emphasized connected meaning. Conversely, focusing processing on surface form-superficial 
characteristics of meaningful stimuli, such as spelling — reduced false memories more in older than younger children (Holliday, Brainerd, \& Reyna, 2011).

Thus, FTT predicts developmental reversals in false memory when these errors involve connecting highly meaningful global gist over superficially distinct elements, as examples, the repeated semantic theme of a list of words or the moral of an entire story containing connected events. Global gist - the theme that connects a list of words or a set of sentences--is not spontaneously extracted in young children (Reyna, 2013). So, the theory makes a subtle Age X False Memory cross-over prediction: Developmental reversals will be obtained for false memories that tap such global gist, but the usual age declines from childhood to adulthood will be obtained for false memories that do not tap global gist, because verbatim memory is also improving during the same age period (Holliday \& Weekes, 2006). The balance of overarching meaning and countervailing verbatim editing, which can be predicted and modeled using FTT, determines the direction of developmental trends.

\section{Conclusions}

The distinguishing features of FTT's account of false memory, features that run throughout this article, are unification and prediction. With respect to unification, the challenge that a theory of false memory must confront is that this is not a narrow laboratory effect but, rather is a broad phenomenon, in two senses: It occurs for a wide range of materials, from word lists, to sentences, to narratives, to everyday experience, and in each of those spheres, it often arises spontaneously. The question is whether one can explain false memory in all its variety with a single set of principles that are not hopelessly complex. Particular interest attaches to whether principles that have been tested with simple materials also work in the complex domains that are foci of applied memory research, Here, we saw, first, that FTT is able to achieve 
unification across materials and paradigms with some very economical idea-basically, that subjects store dissociated verbatim and gist traces of experience that fade at different rates, that access to the two types of traces is controlled by different retrieval cues, and that the two types of traces have contrasting effects on false memory.

Second, a point of particular emphasis in this paper is that these simple ideas provide as much explanatory leverage on complex materials (e.g., narratives, personal experiences that figure in legal testimony) as they do for word lists. In that connection, we reported new findings on false memory for narratives that supplied examples of complex false memory effects that can be explained in the same way as corresponding effects for word lists. Mathematical models revealed the contributions of gist and verbatim processes to false-memory reports for sentences. Thus, the evidence argues against the notion that a separate theory is needed to explain false memory in the domains that are of greatest in applied memory research.

Turning to prediction, readers will have noticed that FTT is proactive; that in addition to explaining established false memory effects with simple principles, emphasis is placed on exploiting those principles to forecast new effects. Several illustrations were discussed in earlier sections: that gist traces are the key ingredient in spontaneous false memories; that false memories will increase over time; and that strong gist traces can induce illusory vivid phenomenology. To supply differential tests of FTT, predictions were featured that are quite counterintuitive from the perspective of other theories, with developmental reversals in false memory and greater long-term stability of false than true memories being cases in point. Importantly for applied memory research, we saw that FTT makes such predictions about complex materials and about the personal experiences that figure in legal testimony. Some examples from the narrative false memory experiment that we reported are that false memory 
reports increase as the similarity between true and false statements increases and that the tendency to correctly accept presented statements is independent of the tendency to erroneously accept true-but-not-presented statements.

This second theme, predictive power, is of special significance in applied research on false memory. In memory for everyday experience, in particular, it is essential to be able to forecast, on the basis of general principles, which situations will produce higher versus lower rates of memory distortion. That is because everyday experience is quite variable, and without predictive principles that cut across such variability, science is unable to say which accounts of events are more likely to be true and which are more likely to be false. For applied memory research, that is unacceptable because the ability to draw such conclusions is often critical, with expert scientific testimony in cases in which different witnesses remember events in contrasting ways being a prime example. 


\section{References}

Alba, J. W., \& Hasher, L. (1983). Is memory schematic?. Psychological Bulletin, 93(2), 203-231.

Barnhardt, T.M., Choi, H., Gerkens, D. R., \& Smith, S. M. (2006). Output position and word relatedness effects in a DRM paradigm: Support for a dual-retrieval process theory of free recall and false memories. Journal of Memory and Language, 55, 213-231.

Bjorklund, D. F. (2014). False-memory creation in children and adults: Theory, research, and implications. Psychology Press.

Brainerd, C. J., \& Mojardin, A. H. (1998). Children's and adults' spontaneous false memories: Long- term persistence and mere- testing effects. Child Development, 69(5), 1361-1377.

Brainerd, C. J., \& Reyna, V. F. (1992). Explaining “memory free” reasoning. Psychological Science, 3(6), 332-339.

Brainerd, C. J., \& Reyna, V. F. (2002). Fuzzy-trace theory and false memory. Current Directions in Psychological Science, 11(5), 164-169.

Brainerd, C. J., \& Reyna, V. F. (2005). The science of false memory. Oxford University Press.

Brainerd, C. J., \& Reyna, V. F. (2008). Episodic over-distribution: A signature effect of familiarity without recollection. Journal of Memory and Language, 58(3), 765-786.

Brainerd, C. J., Reyna, V. F., \& Estrada, S. (2006). Recollection rejection of false narrative statements. Memory, 14(6), 672-691.

Brainerd, C. J., Reyna, V. F., \& Forrest, T. J. (2002). Are young children susceptible to the falsememory illusion?. Child Development, 73(5), 1363-1377.

Brainerd, C. J., Reyna, V. F., Holliday, R. E., \& Nakamura, K. (2012). Overdistribution in source memory. Journal of Experimental Psychology: Learning, Memory, and Cognition, 38(2), 413. 
Brainerd, C. J., Reyna, V. F., \& Howe, M. L. (2009). Trichotomous processes in early memory development, aging, and neurocognitive impairment: A unified theory. Psychological Review, $116(4), 783-832$.

Brainerd, C. J., Reyna, V. F., \& Kneer, R. (1995). False-recognition reversal: When similarity is distinctive. Journal of Memory and Language, 34(2), 157-185.

Brainerd, C. J., Reyna, V. F., Wright, R., \& Mojardin, A. H. (2003). Recollection rejection: false-memory editing in children and adults. Psychological Review, 110(4), 762-784.

Brainerd, C. J., Reyna, V. F., \& Zember, E. (2011). Theoretical and forensic implications of developmental studies of the DRM illusion. Memory \& Cognition, 39, 365-380. doi:10.3758/s13421-010-0043-2

Brainerd, C. J., Wright, R., Reyna, V. F., \& Mojardin, A. H. (2001). Conjoint recognition and phantom recollection. Journal of Experimental Psychology: Learning, Memory, and Cognition, 27(2), 307-327.

Brainerd, C. J., Yang, Y., Reyna, V. F., Howe, M. L., \& Mills, B. A. (2008). Semantic processing in "associative" false memory. Psychonomic Bulletin \& Review, 15(6), 1035-1053.

Bransford, J. D., \& Franks, J. J. (1971). The abstraction of linguistic ideas. Cognitive Psychology, 2(4), 331-350.

Britt, A., Kurby, C. A., Dandotkar, S., Wolfe, C. R. (2008). I agreed with what? Memory for simple argument claims. Discourse Processes, 45(1), 52-84.

Cann, D. R., McRae, K., \& Katz, A. N. (2011). False recall in the Deese-Roediger-McDermott paradigm: The roles of gist and associative strength. The Quarterly Journal of Experimental Psychology, 64(8), 1515-1542. 
Ceci, S. J., \& Bruck, M. (1993). Suggestibility of the child witness: A historical review and synthesis. Psychological Bulletin, 113(3), 403-439.

Ceci, S. J., Papierno, P. B., \& Kulkofsky, S. (2007). Representational constraints on children's suggestibility. Psychological Science, 18(6), 503-509.

Clark, H. H., \& Clark, E. V. (1977). Psychology and language. New York: Harcourt Brace Jovanovich.

Deese, J. (1959). On the prediction of occurrence of particular verbal intrusions in immediate recall. Journal of Experimental Psychology, 58(1), 17-22.

Dewhurst, S. A., Pursglove, R. C., \& Lewis, C. (2007). Story contexts increase susceptibility to the DRM illusion in 5-year-olds. Developmental Science, 10, 374-378. http://dx.doi.org/10.1111/j.1467-7687.2007.00592.x.

Dobbins, I. G., Kroll, N. E., \& Liu, Q. (1998). Confidence-accuracy inversions in scene recognition: A remember-know analysis. Journal of Experimental Psychology: Learning, Memory, and Cognition, 24(5), 1306-1315.

Dodson, C. S., \& Shimamura, A. P. (2000). Differential effects of cue dependency on item and source memory. Journal of Experimental Psychology: Learning, Memory, and Cognition, 26(4), 1023-1044.

Faigman, D. L., \& Monahan, J. (2005). Psychological evidence at the dawn of the law's scientific age. Annual Review of Psychology, 56, 631-659.

Fisher, R. P., \& Chandler, C. C. (1991). Independence between recalling interevent relations and specific events. Journal of Experimental Psychology: Learning, Memory, \& Cognition, 17, $722-733$. 
Fischer, G. W., \& Hawkins, S. A. (1993). Strategy compatibility, scale compatibility, and the prominence effect. Journal of Experimental Psychology: Human Perception and Performance, 19(3), 580-597.

Frenda, S. J., Nichols, R. M., \& Loftus, E. F. (2011). Current issues and advances in misinformation research. Current Directions in Psychological Science, 20(1), 20-23.

Gallo, D. A. (2006). Associative illusions of memory: False memory research in DRM and related tasks. New York: Psychology Press.

Gallo, D. A. (2010). False memories and fantastic beliefs: 15 years of the DRM illusion. Memory \& Cognition, 38(7), 833-848.

Gallo, D. A., \& Roediger, H. L. (2003). Variability among word lists in eliciting memory illusions: evidence for associative activation and monitoring. Journal of Memory and Language, 47, 469-497.

Garner, S. R., \& Howe, M. L. (2014). False memories from survival processing make better primes for problem-solving, Memory, 22(1), 9-18. doi: 10.1080/09658211.2012.759975.

Gerkens, D. R., \& Smith, S. M. (2004). Shifting modality between study and test: A fuzzy-trace theory analysis. Psychonomic Bulletin and Review, 11, 143-149.

Ghetti, S. (2008). Processes underlying developmental reversals in false-memory formation: comment on Brainerd, Reyna, and Ceci (2008). Psychological Bulletin, 134(5), 764-767.

Glucksberg, S., \& Danks, J. H. (1975). Experimental psycholinguistics: An introduction. Lawrence Erlbaum.

Goldsmith, M., Koriat, A., \& Pansky, A. (2005). Strategic regulation of grain size in memory reporting over time. Journal of Memory and Language, 52, 505-525. 
Hans, V. P., \& Reyna, V. F. (2011). To dollars from sense: Qualitative to quantitative translation in jury damage awards. Journal of Empirical Legal Studies, 8(s1), 120-147.

Holliday, R. E., Brainerd, C. J., \& Reyna, V. F. (2008). Recall of details never experienced: Effects of age, repetition, and semantic cues. Cognitive Development, 23, 67-78. http://dx.doi.org/10.1016/j.cogdev.2007.05.002.

Holliday, R. E., Brainerd, C. J., \& Reyna, V. F. (2011). Developmental reversals in false memory: Now you see them, now you don't. Developmental Psychology, 47, 442-449. http://dx.doi.org/10.1037/a0021058.

Holliday, R. E., \& Weekes, B. S. (2006). Dissociated developmental trajectories for semantic and phonological false memories. Memory, 14, 624-636.

Howe, M.L. (2008) What is false memory development the development of? Comment on Brainerd, Reyna, and Ceci (2008). Psychological Bulletin, 134, 768-772. doi: 10.1037/00332909.134.5.768

Howe, M. L., \& Wilkinson, S. (2011). Using story contexts to bias children's true and false memories. Journal of Experimental Child Psychology, 108, 75-95. http://dx.doi.org/10.1016/j.jecp. 2010.06.009.

Huff, M. J., McNabb, J. \& Hutchinson, K. A. (2015). List blocking and longer retention intervals reveal an influence of gist processing for lexically ambiguous critical lures. Memory and Cognition, 43, 1193-1207.

Hunt, R.R., \& Ellis, H.C. (2004). Fundamentals of cognitive psychology, 7th edition. Boston: McGraw-Hill 
Hutchison, K. A., \& Balota, D. A. (2005). Decoupling semantic and associative information in false memories: Explorations with semantically ambiguous and unambiguous critical lures. Journal of Memory and Language, 52(1), 1-28.

Johnson, M. K., Hashtroudi, S., \& Lindsay, D. S. (1993). Source monitoring. Psychological Bulletin, 114, 3-28.

Kintsch, W. (1975).The representation of meaning in memory. Hillsdale, NJ: Erlbaum.

Keren, G., \& Schul, Y. (2009). Two is not always better than one: A critical evaluation of two-system theories. Perspectives On Psychological Science, 4(6), 533-550. doi:10.1111/j.17456924.2009.01164.x

Klaczynski, P.A. (2009). Cognitive and social cognitive development: Dual-process research and theory. In J. T. Evans \& K. Frankish (Eds.), In two minds: Dual processes and beyond (pp. 265-292). Oxford University Press; New York, NY.

Liben, L. S. (1977). Memory in the context of cognitive development: The Piagetian approach. In R. V. Kail Jr., \& J. W. Hagen (Eds.), Perspectives on the development of memory and cognition (pp. 297-331). Hillsdale, NJ: Lawrence Erlbaum Associates.

Lim, P. L. (1993). Meaning versus verbatim memory in language processing: Deriving inferential, morphological, and metaphorical gist. (Doctoral dissertation). Retrieved from http://arizona.openrepository.com/arizona/handle/10150/186487

Lloyd, F. J., \& Reyna, V. F. (2009). Clinical gist and medical education: Connecting the dots. JAMA, 302(12), 1332-1333.

Mandler, G. (1962). From association to structure. Psychological Review, 69, 415-427.

Mayer, R. E. (2002). Rote versus meaningful learning. Theory Into Practice, 41(4), 226-232. 
McElroy, T., Dickinson, C., Corbin, J., \& Beck, H. (2015). Tracking risky decisions: Comparing fuzzy-trace theory and prospect theory through eye-tracking. Manuscript submitted for publication.

Meade, M. L., Watson, J. M., Balota, D. A., \& Roediger, H.L. (2007). The roles of spreading activation and retrieval mode in producing false recognition in the DRM paradigm. Journal of Memory and Language, 56, 305-320.

Michael, E. B., Allison, T., Danks, J. H., de Terra, D., Massaro, D., \& Donavos, D. (2007). Does verbatim translation help summary translation? Proceedings of the 6th International Symposium on Bilingualism, Hamburg, Germany.

Mojardín, H. A. (1998). The underlying memory processes of adults spontaneous and implanted false memories. Unpublished doctoral dissertation.

Nelson, D. L., McEvoy, C. L., \& Schreiber, T. A. (2004). The University of South Florida free association, rhyme, and word fragment norms. Behavior Research Methods, Instruments, \& Computers, 36(3), 402-407.

New, J. J., \& German, T. C. (2014). Spiders at the cocktail party: An ancestral threat that surmounts inattentional blindness. Evolution and Human Behavior, 36(3), 165-173. doi:10.1016/j.evolhumbehav.2014.08.004

Ost, J., Blank, H., Davies, J., Jones, G., Lambert, K., \& Salmon, K. (2013). False memorył false memory: DRM errors are unrelated to the misinformation effect. PloS one, 8(4), e57939.

Paivio, A. (1971). Imagery and verbal processes. Oxford, England: Holt, Rinehart \& Winston.

Pansky, A., \& Tenenboim, E. (2011). Inoculating against eyewitness suggestibility via interpolated verbatim vs. gist testing. Memory \& Cognition, 39(1), 155-170. 
Pennington, N., \& Hastie, R. (1992). Explaining the evidence: Tests of the story model for juror decision making. Journal of Personality and Social Psychology, 62(2), 189-206.

Reyna, V. F. (2008). A theory of medical decision making and health: Fuzzy-trace theory. Medical Decision Making, 28(6), 850-865.

Reyna, V. F. (2012). A new intuitionism: Meaning, memory, and development in fuzzy-trace theory. Judgment \& Decision Making, 7(3), 332-359.

Reyna, V. F. (2013). Intuition, reasoning, and development: A fuzzy-trace theory approach. In P. Barrouillet \& C. Gauffroy (Eds.), The development of thinking and reasoning (pp.193-220). Hove, UK: Psychology Press.

Reyna, V. F., \& Brainerd, C. J. (1995a). Fuzzy-trace theory: An interim synthesis. Learning and Individual Differences, 7(1), 1-75.

Reyna, V. F., \& Brainerd, C. J. (1995b). Fuzzy-trace theory: Some foundational issues. Learning and Individual Differences, 7(2), 145-162.

Reyna, V. F., \& Brainerd, C. J. (1998). Fuzzy-trace theory and false memory: New frontiers. Journal of Experimental Child Psychology, 71(2), 194-209.

Reyna, V. F., \& Brainerd, C. J. (2011). Dual processes in decision making and developmental neuroscience: A fuzzy-trace model. Developmental Review, 31(2), 180-206.

Reyna, V. F., Holliday, R., \& Marche, T. (2002). Explaining the development of false memories. Developmental Review, 22(3), 436-489.

Reyna, V. F., \& Kiernan, B. (1994). Development of gist versus verbatim memory in sentence recognition: Effects of lexical familiarity, semantic content, encoding instructions, and retention interval. Developmental Psychology, 30(2), 178-191. doi:10.1037/00121649.30.2.178 
Reyna, V. F., \& Kiernan, B. (1995). Children's memory and metaphorical interpretation. Metaphor and Symbol, 10(4), 309-331.

Reyna, V. F., \& Lloyd, F. (1997). Theories of false memory in children and adults. Learning and Individual Differences, 9(2), 95-123.

Reyna, V. F., Lloyd, F. J., \& Brainerd, C. J. (2003). Memory, development, and rationality: An integrative theory of judgment and decision making. In S. Schneider \& J. Shanteau (Eds.), Emerging perspectives on judgment and decision research (pp. 201-245). New York:

Cambridge University Press.

Reyna, V. F., \& Mills, B. A. (2007). Interference processes in fuzzy-trace theory: Aging, Alzheimer's disease, and development. In C. MacLeod \& D. Gorfein (Eds.), Inhibition in cognition (pp. 185-210). Washington: APA Press.

Reyna, V. F., Mills, B. A., Estrada, S. M., \& Brainerd, C. J. (2007). False memory in children: Data, theory, and legal implications. In M. P. Toglia, J. D. Read, D. F. Ross, \& R. C. L. Lindsay (Eds.), The handbook of eyewitness psychology: Memory for events (pp. 473-510). Mahwah, NJ: Erlbaum.

Reyna, V. F., \& Titcomb, A. L. (1997). Constraints on the suggestibility of eyewitness testimony: A fuzzy-trace theory analysis. In D. G. Payne \& F. G. Conrad (Eds.), A synthesis of basic and applied approaches to human memory (pp. 157-174). Hillsdale, NJ: Erlbaum.

Reyna, V. F., Wilhelms, E. A., McCormick, M. J., \& Weldon, R. B. (2015). Development of risky decision making: Fuzzy-trace theory and neurobiological perspectives. Child Development Perspectives, 9(2), 122-127. doi: 10.1111/cdep.12117.

Robinson, K. J., \& Roediger, H. L. (1997). Associative processes in false recall and false recognition. Psychological Science, 8(3), 231-237. 
Roediger, H.L. \& Karpicke, J.D. (2006). Test-enhanced learning: Taking memory tests improves long-term retention. Psychological Science, 17, 249-255.

Roediger, H. L., III, \& McDermott, K. B. (1995). Creating false memories: Remembering words not presented in lists. Journal of Experimental Psychology: Learning, Memory, \& Cognition, $21,803-814$.

Roediger, H. L., Watson, J. M., McDermott, K. B., \& Gallo, D. A. (2001). Factors that determine false recall: A multiple regression analysis. Psychonomic Bulletin \& Review, 8(3), 385-407.

Schacter, D. L., Israel, L., \& Racine, C. (1999). Suppressing false recognition in younger and older adults: The distinctiveness heuristic. Journal of Memory and Language, 40(1), 1-24.

Schacter, D. L., Guerin, S. A., \& St. Jacques, P.L. (2012). Memory distortion: An adaptive perspective. Trends in Cognitive Science, 15 (10), 467-474.

Schneider, D. W., \& Anderson. J. R. (2012). Modeling fan effects on the time course of associative recognition. Cognitive Psychology, 64 (3), 127-160

Singer, M., \& Remillard, G. (2008). Veridical and false memory for text: A multiprocess analysis. Journal of Memory and Language, 59(1), 18-35.

Spiro, R. J. (1980). Constructive processes in prose comprehension and recall. In R.J. Spiro, B.C. Bruce, \& W.F. Brewer (Eds.), Theoretical issues in reading comprehension (pp. 245-278). Hillsdale, NJ: Lawrence Erlbaum Associates.

Stadler, M. A., Roediger, H. L., \& McDermott, K. B. (1999). Norms for word lists that create false memories. Memory \& Cognition, 27(3), 494-500.

Stahl, C., \& Klauer, K. C. (2008). A simplified conjoint recognition paradigm for the measurement of gist and verbatim memory. Journal of Experimental Psychology: Learning, Memory, and Cognition, 34(3), 570-586. 
Thapar, A., \& McDermott, K. B. (2001). False recall and false recognition induced by presentation of associated words: Effects of retention interval and level of processing. Memory \& Cognition, 29(3), 424-432.

Toglia, M. P. (1999). Recall accuracy and illusory memories: When more is less. Memory, 7(2), $233-256$.

Tolman, E. C. (1938). The determiners of behavior at a choice point. Psychological Review, 45(1), 1-41.

Tolman, E. C. (1948). Cognitive maps in rats and men. Psychological Review, 55(4), 189-208.

Underwood, B. J. (1965). False recognition produced by implicit verbal responses. Journal of Experimental Psychology, 70(1), 122-129. 


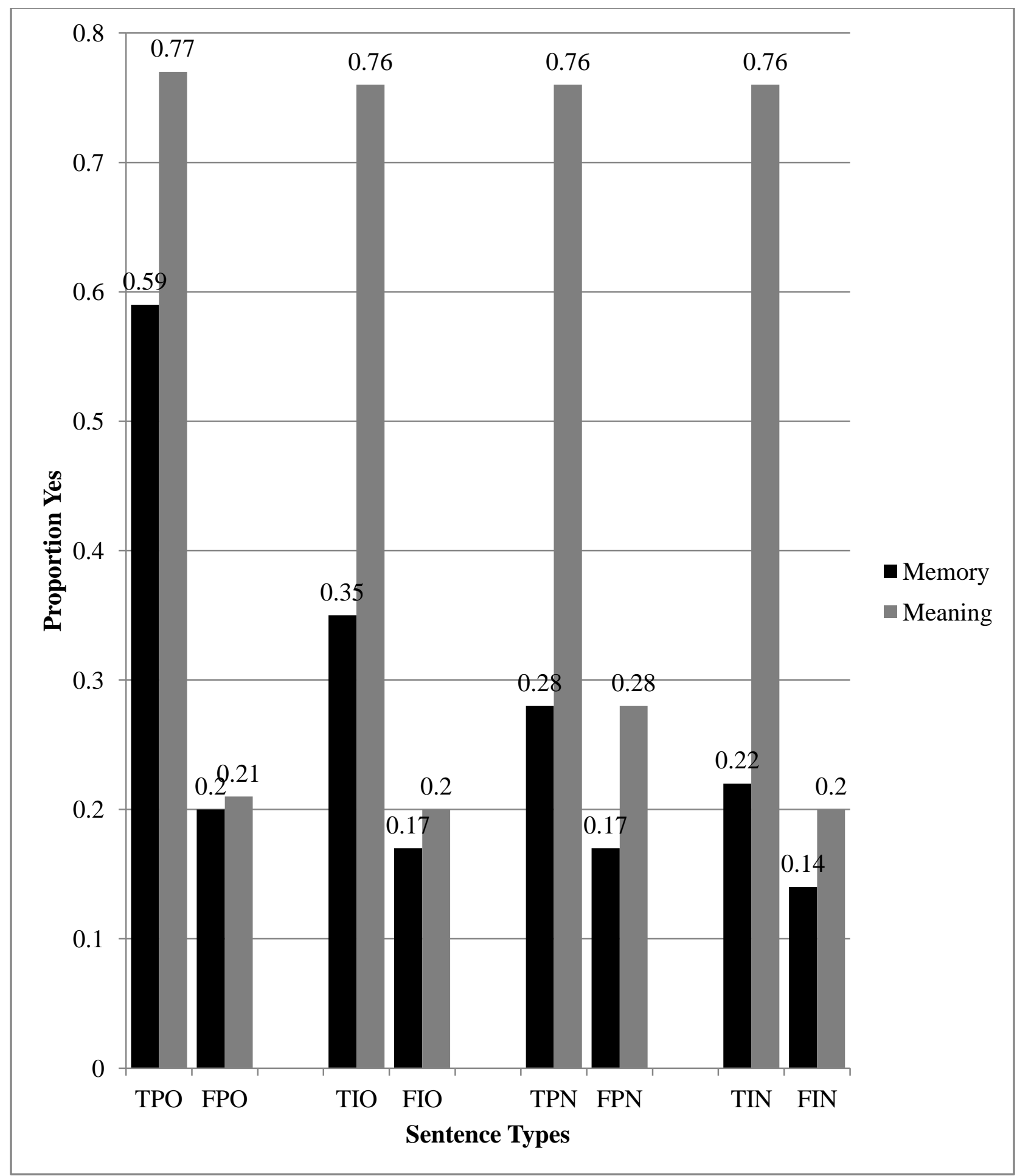

Figure 1. Proportion "yes" responses for different sentence types. TPO $=$ True premise with original wording; TPN $=$ True premise with novel wording; TIO $=$ True inference with original wording; TIN $=$ True inference with novel wording; FPO $=$ False premise with original wording; FPN $=$ False premise with novel wording; $F 1 O=$ False inference with original wording; FIN = False inference with novel wording. 